\title{
Progressive Relapse of Ligamentum Flavum Ossification Following Decompressive Surgery
}

\author{
Kei Ando, Shiro Imagama, Zenya Ito, Kazuyoshi Kobayashi, Junichi Ukai, Akio Muramoto, \\ Ryuichi Shinjo, Tomohiro Matsumoto, Hiroaki Nakashima, Naoki Ishiguro \\ Department of Orthopaedic Surgery, Nagoya University Graduate School of Medicine, Nagoya, Japan
}

Thoracic ossification of the ligamentum flavum (T-OLF) is a relatively rare spinal disorder that generally requires surgical intervention, due to its progressive nature and the poor response to conservative therapy. The prevalence of OLF has been reported at $3.8 \%-26 \%$, which is similar to that of cervical ossification of the posterior longitudinal ligament (OPLL). The progression of OPLL after cervical laminoplasty for the treatment of OPLL is often shown in long-term follow-up. However, there have been no reports on the progression of OLF following surgery. We report a case of thoracic myelopathy secondary to the progressive relapse of OLF following laminectomy.

Keywords: Thoracic ossification of ligamentum flavum; Progressive relapse

\section{Introduction}

Thoracic ossification of the ligamentum flavum (T-OLF) frequently occurs at multiple segments of the thoracic spine, particularly at the lower levels. Although there are reports on the etiology and pathogenesis of this disease, its main causes remain unclear. Moreover, there are no reports on T-OLF changes following posterior laminectomy and removal of the OLF. However, the morphological changes that occur with cervical ossification of the posterior longitudinal ligament (OPLL) following laminoplasty have been reported [1-4]. We report a case of salvage surgery for relapsed thoracic myelopathy secondary to the progressive relapse of OLF following laminectomy.

\section{Case Report}

Seven years ago, a 36-year-old man presented at another institution with gradually progressive difficulty in walking and numbness in both lower extremities for six months. He underwent T3-5 laminectomy for OLF-induced myelopathy and L2-4 postero-lateral fusion with instrumentation for canal stenosis (Fig. 1). He was symptom free for five years. When the symptoms started again and increased in intensity, the patient was referred to our institution for further management. On admission, the preoperative examination revealed grade 4 muscle strength and hypoesthesia below at the T7 area. He could perform the one-leg standing test on each leg, but only for two seconds. He was hyperreflexive in both lower limbs with a bilateral Babinski response. His preoperative Japanese Orthopaedic Association score (JOA score) was 12 (4, 2, $2,0,1,3)$ in cervical and $6(2,0,1,3)$ in thoracic. The patient had a height of $183 \mathrm{~cm}$ and weight of $122 \mathrm{~kg}$; and his age-adjusted body mass index was $36.4 \mathrm{~kg} / \mathrm{m}^{2}$.

Plain radiographs of the spine showed $47^{\circ}$ thoracic

Received Nov 27, 2013; Revised Jan 1, 2014; Accepted Jan 24, 2014

Corresponding author: Shiro Imagama

Department of Orthopaedic Surgery, Nagoya Graduate University School of Medicine,

65 Tsurumai Showa-ward, Aichi 466-8550, Japan

Tel: +81-52-833-6021, Fax: +81-52-833-6021, E-mail: imagama@med.nagoya-u.ac.jp 
kyphosis (T1-12), $26^{\circ}$ thoracolumbar kyphosis (T10-L2) and $8.6 \mathrm{~cm}$ of sagittal vertical axis (SVA) (Fig. 2A). Reconstructed computed tomography (CT) showed OPLL from $\mathrm{C} 2-5$ and T3-6 (Fig. 2B). The spinal cord was compressed by OLF at T1-3 and T7-11 (Fig. 2C). The cervical cord was also compressed moderately at multiple levels. We performed C3-7 laminoplasty (Fig. 3A), T1-11 laminectomy and T6-9 posterior fusion with instrumentation, as there were multiple levels with severe OLF. Also more than half of the facets at the kyphotic thoracic spine needed facetectomy, although mobile segments were to be preserved with a fused L2-4 (Fig. 3B). After surgery, his symptoms disappeared except for slight numbness in legs, and the patient returned to work.

Eleven months after the surgery, the patient presented with severe pain in the lower back, numbness in both legs and gait disturbance. He had been asymptomatic until that time. His JOA score had deteriorated to $8(3,1,2,2)$ in cervical and $3(1,0,0,2)$ in thoracic. On radiological evaluation, thoracolumbar kyphosis had progressed from
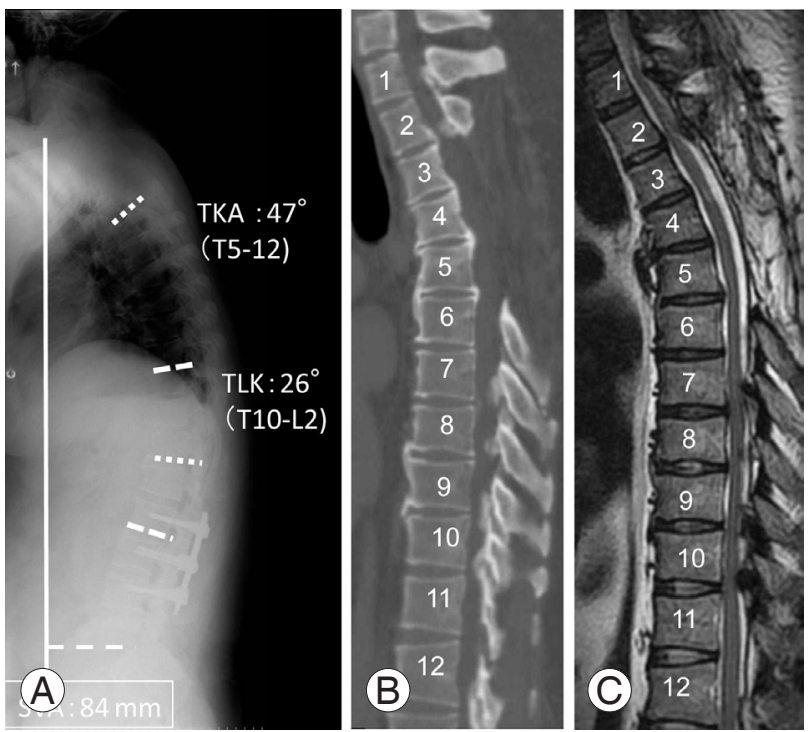

Fig. 2. (A) Radiograph of the spine showing $47^{\circ}$ thoracic kyphosis (TKA, T1-12), 26 thoracolumbar kyphosis (TLK, T10-L2) and $84 \mathrm{~mm}$ of sagittal vertical axis (SVA). (B, C) Computed tomography and magnetic resonance imaging showing ossification of the posterior longitudinal ligament from $\mathrm{C} 2$ to $\mathrm{C} 5$ and $\mathrm{T} 3$ to T6. The spinal cord was compressed by the ossification of the ligamentum flavum at T1-3 and T7-11.
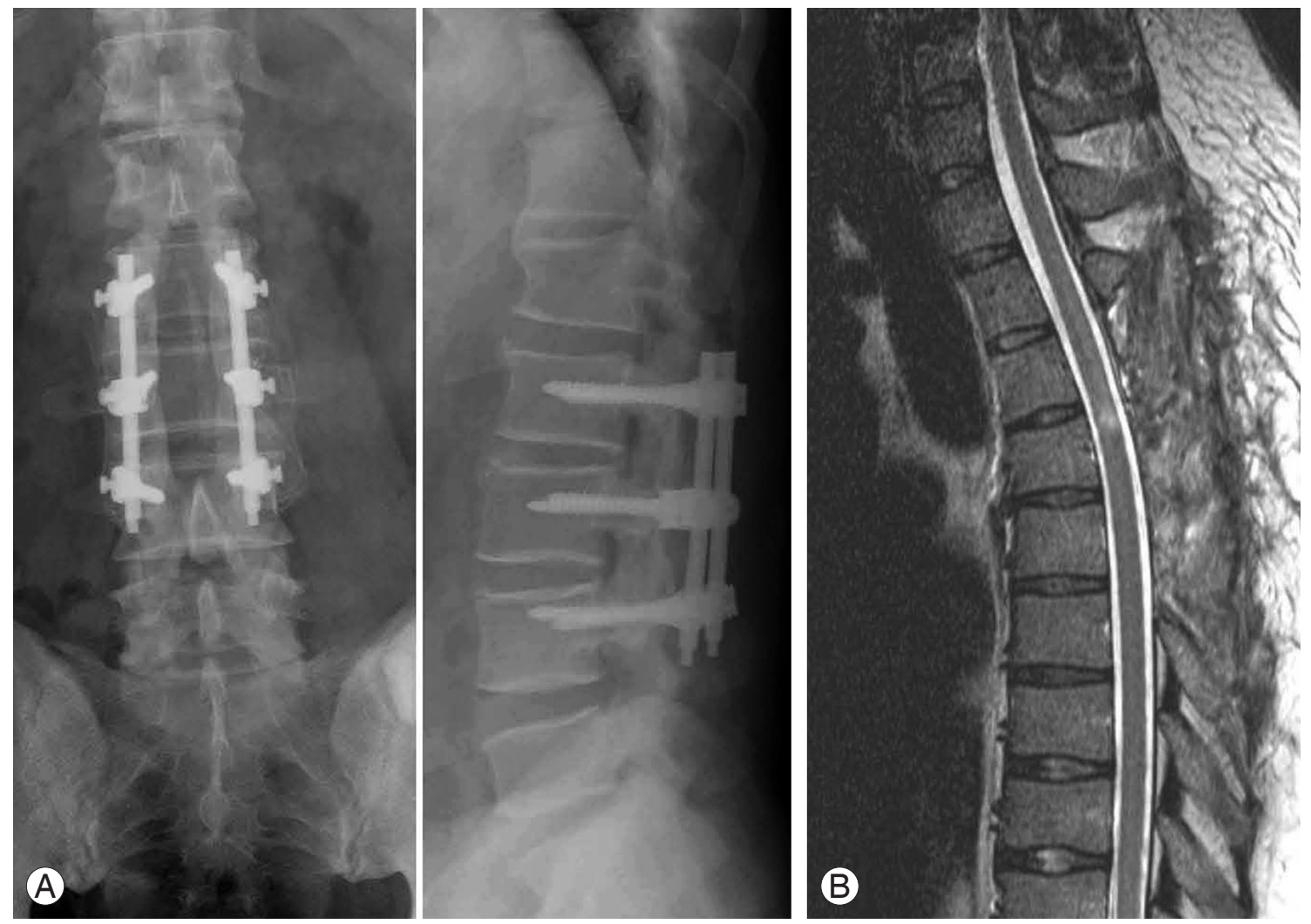

Fig. 1. (A) Radiograph of anteroposterior and lateral views of lumbar spine demonstrating posterolateral fusion of L2-4. Postoperative magnetic resonance imaging from another institution. (B) The spinal cord is decompressed sufficiently with no evidence of ossification in the thoracic spine (from seven years ago). 
$26^{\circ}$ to $39^{\circ}$ (Fig. $4 \mathrm{~A}$ ). MRI revealed a severely compressed spinal cord with high intensity areas at the T10-11 level (Fig. 4B). CT showed relapsed ossification: a small ossification medial to the T11 superior articular process, extending to the inferior articular process of T10 and along the dura mater at that same level (Fig. 4B). Considering the situation, we performed a revision surgery, wherein we fused from T6 to L4 with instrumentation and removed the propagated ossification at T10/11 using navigation. However, due to the ossification of the dura mater, we were unable to completely resect the OLF, and we allowed some areas to float. We confirmed decompression of spinal cord by echo imaging. The postoperative course was uneventful, and his neurologic improvements were excellent with relief of low back pain. The patient could walk on the fifth postoperative day. Two years after the last surgery, the patient is pain free and walking comfortably without support, and his JOA score is $9(3,1,2,3)$. Follow-up radiographs and $\mathrm{CT}$ at that time showed bony union within the instrumented levels (Fig. 5).
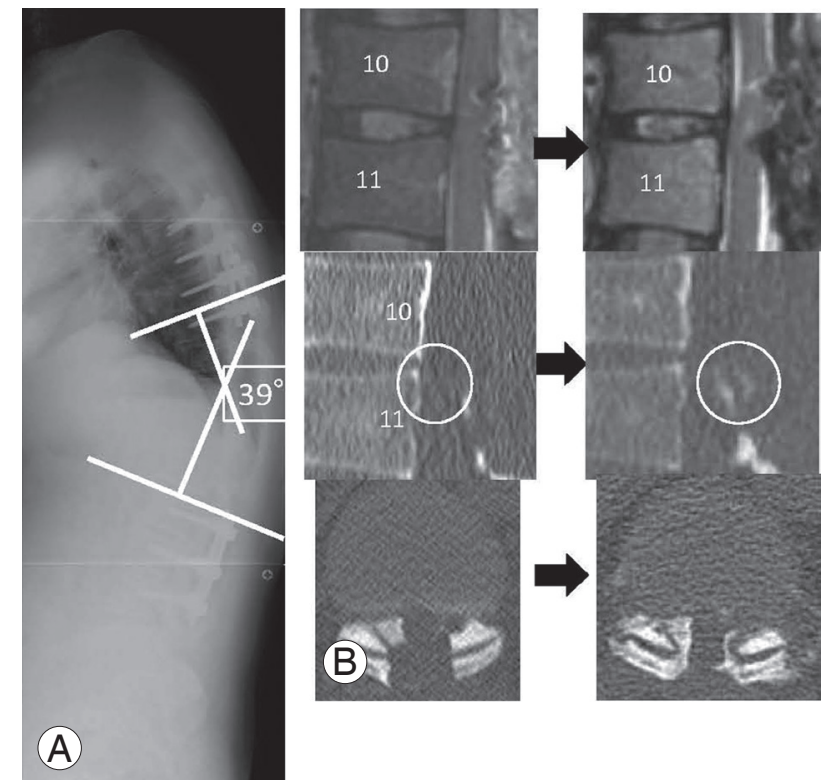

Fig. 4. (A) Thoracolumbar kyphosis progressed from $25^{\circ}$ to $39^{\circ}$. (B) Computed tomography showing relapsed ossification from a small ossification medial to the T11 superior articular process, extending to the inferior articular process of $\mathrm{T} 10$ and along the dura mater at the same level.

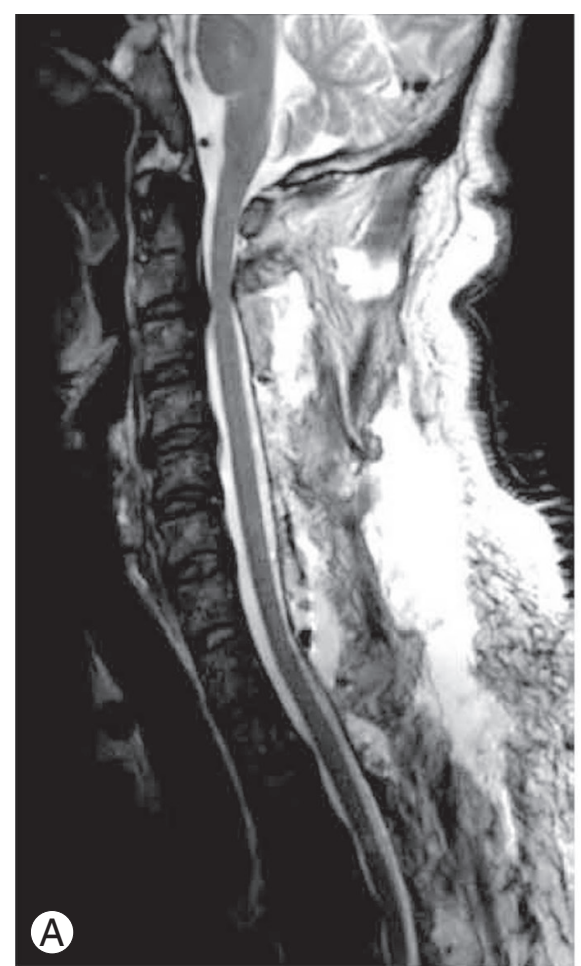

Fig. 3. (A) Magnetic resonance imaging after cervical laminoplasty. (B) Radiographs after T1-11 laminectomy and T6-9 posterior fusion with instrumentation.
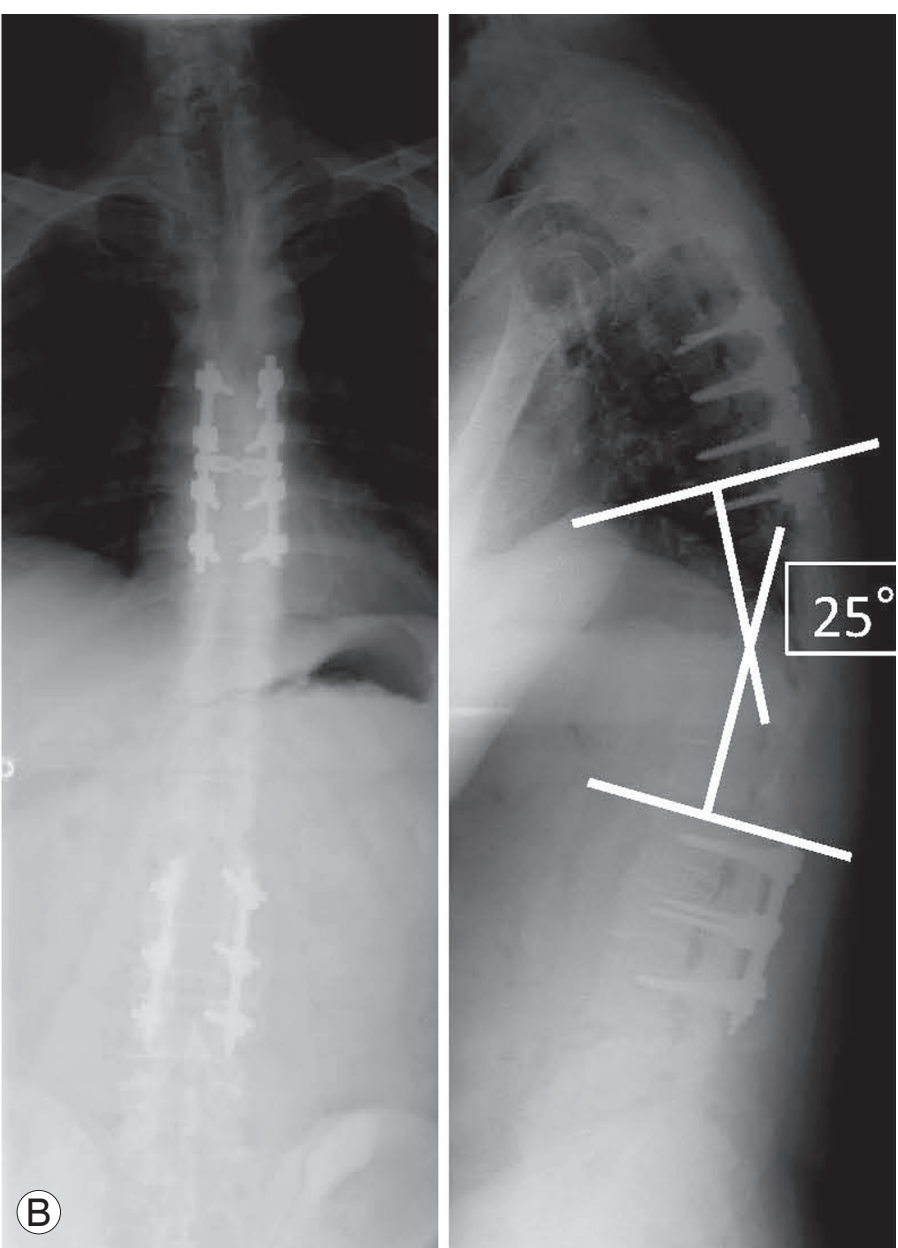


\section{Discussion}

OPLL and OLF are recognized as common multifactorial diseases in Japan $[5,6]$. OLF frequently occurs at lower thoracic levels, because there is increased mechanical stress where the thoracic vertebrae form the junction between the rigid rib cage and the elastic lumbar spine. There is a direct correlation between increased mobility of the spine with repetitive mild trauma and the high

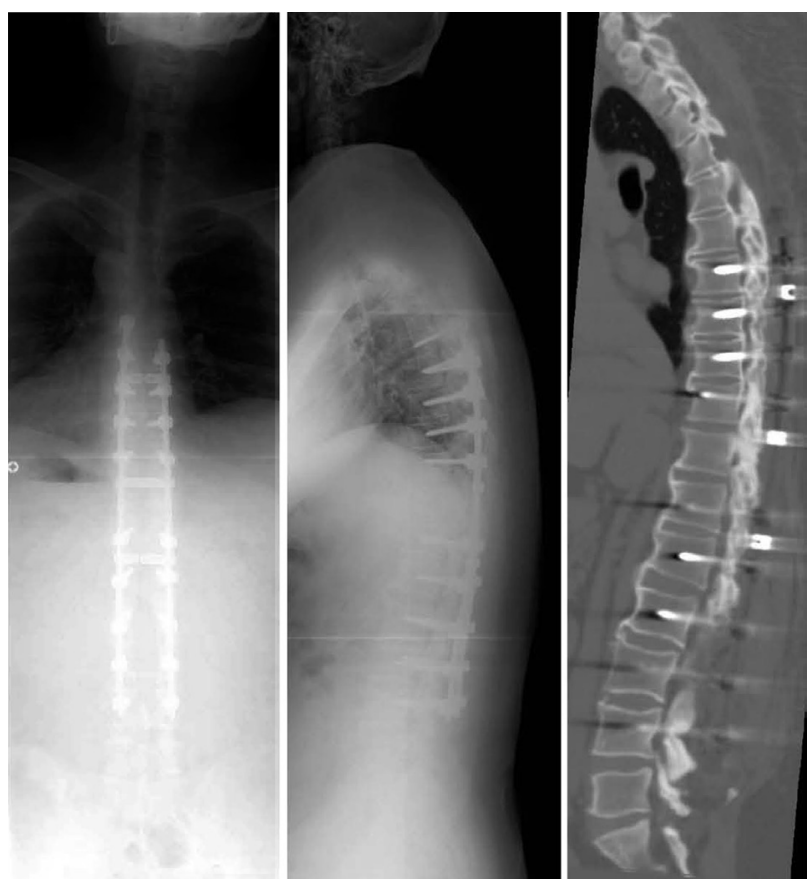

Fig. 5. Radiograph and computed tomography (two years after surgery) shows bony union within the instrumented levels. tensile force present in the posterior column [7]. The main reason for the multiple areas of T-OLF in this case was likely due to the increased mechanical stress at the levels rostral and caudal to the fused continuous OPLL, extending from $\mathrm{T} 3$ to $\mathrm{T} 6$, following laminectomy at the first institution (Fig. 6A). Although there have been some reports on progressive ossification following laminoplasty with cervical OPLL [1-4], we could not find any reports on progressive thoracic ossification. Ha et al. [8] has reported on thoracic myelopathy caused by OLF in patients with posterior instrumented lumbar fusion, with pathogenesis similar to the present case.

The patient in this case was considered for three surgical strategies. The first was decompression of the thoracic segments only without fusion, which could preserve the mobile segments. However, there are some risks with this procedure, such as progressive thoracic kyphosis with $8.6 \mathrm{~cm}$ of SVA and propagated ossification, due to increased stress at the decompression levels under upper thoracic vertebrae with continuous OPLL. The second was a long fusion of the lumbar spine, which did not increase the thoracic kyphosis or propagate ossification in the fused levels (although the patient was 36 years old). The third strategy chosen was T1-11 laminectomy and T6-9 posterior fusion with instrumentation, as there were multiple levels of severe OLF. More than half of the facets at the kyphotic thoracic spine required facetectomy to decompress the spinal cord safely, although mobile segments should be preserved with an L2-4 fusion (Fig. 6B). We considered extending the instrumentation to T11, because of the severe OLF at T10/11. This was not
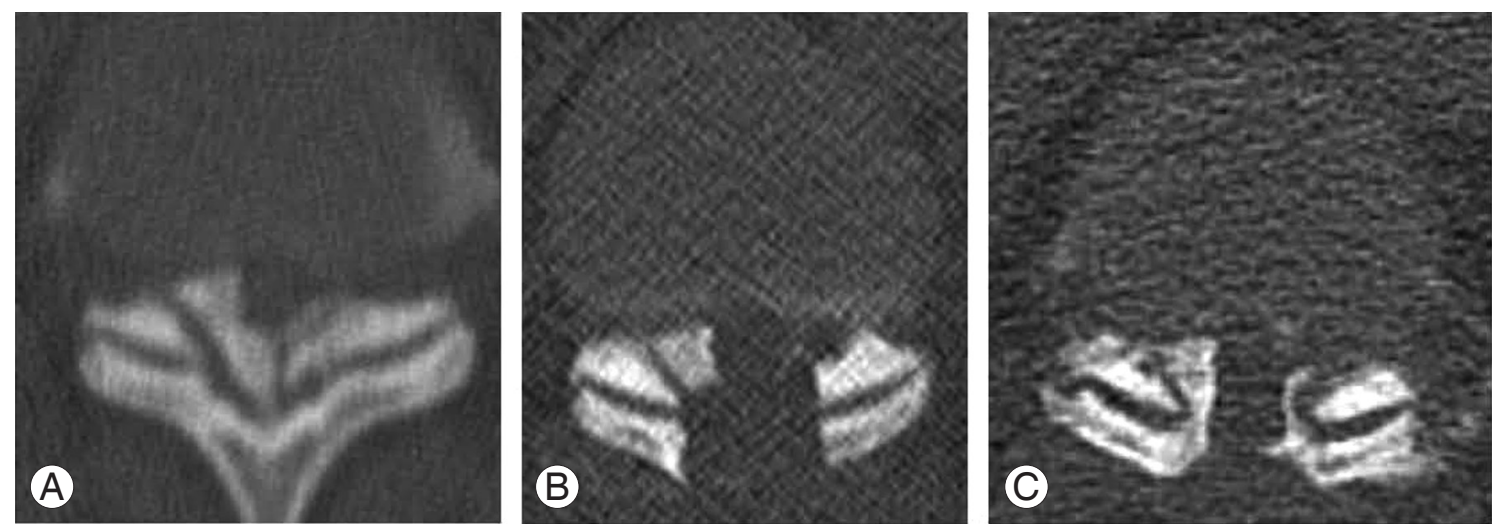

Fig. 6. Progressive relapse of the ossification of the ligamentum flavum at T10-T11. (A) Preoperative view of severely ossified ligamentum flavum. (B) Postoperative view following the first operation at our institution. (C) Relapsed ossification from a small ossification medial to the T11 superior articular process, extending to the inferior articular process of T10 and along the dura mater at the same level. 
done, because we thought problems would occur at the adjacent levels if there were only two mobile segments at the OALL-induced continuous T12-L1 lesion. The OLF that occurred at T10/11, after the first surgery at our institution, may have occurred secondary to the focusing of mechanical stress between the fused segments, which were present both rostrally and caudally (Fig. 6C). Furthermore, the thoracic myelopathy worsened with progression of OLF and the addition of micromotion on the vulnerable spinal cord.

Surgical technique in the salvage procedure for the relapsed OLF was extremely difficult under severe adhesion after the previous decompression and fusion, because of the lack of normal lamina and the presence of ossified dura mater. Therefore, a floating technique was necessary even with the procedure performed under navigation following registration to the costal process of T10. In retrospect, long fusion with instrumentation should be considered for some patients with thoracic OPLL, even in those of relatively young age. We must continue to follow this case carefully for radiological changes, especially changes in the caudal adjacent area.

The current case demonstrated the need for posterior reoperation due to a relapsed OLF following laminectomies. Long fusion with instrumentation should be considered for some patients with thoracic OPLL, even for those of young age.

\section{Conflict of Interest}

No potential conflict of interest relevant to this article was reported.

\section{References}

1. Hori T, Kawaguchi Y, Kimura T. How does the os- sification area of the posterior longitudinal ligament progress after cervical laminoplasty? Spine (Phila Pa 1976) 2006;31:2807-12.

2. Hori T, Kawaguchi Y, Kimura T. How does the ossification area of the posterior longitudinal ligament thicken following cervical laminoplasty? Spine (Phila Pa 1976) 2007;32:E551-6.

3. Murakami M, Seichi A, Chikuda H, Takeshita K, Nakamura K, Kimura A. Long-term follow-up of the progression of ossification of the posterior longitudinal ligament. J Neurosurg Spine 2010;12:577-9.

4. Tokuhashi Y, Ajiro Y, Umezawa N. A patient with two re-surgeries for delayed myelopathy due to progression of ossification of the posterior longitudinal ligaments after cervical laminoplasty. Spine (Phila Pa 1976) 2009;34:E101-5.

5. Inamasu J, Guiot BH, Sachs DC. Ossification of the posterior longitudinal ligament: an update on its biology, epidemiology, and natural history. Neurosurgery 2006;58:1027-39.

6. Ando K, Imagama S, Wakao N, et al. Examination of the influence of ossification of the anterior longitudinal ligament on symptom progression and surgical outcome of ossification of the thoracic ligamentum flavum: a multicenter study. J Neurosurg Spine 2012; 16:147-53.

7. Ando K, Imagama S, Ito Z, et al. Predictive factors for a poor surgical outcome with thoracic ossification of the ligamentum flavum by multivariate analysis: a multicenter study. Spine (Phila Pa 1976) 2013 Mar 7 [Epub]. http://dx.doi.org/10.1097/BRS. 0b013e31828ff736.

8. Ha KY, Seo JY, Son IN, Kim YH, Kim KW. Thoracic myelopathy caused by ossification of the yellow ligament in patients with posterior instrumented lumbar fusion. Eur Spine J 2012;21:2443-9. 